\title{
Proximal Tubulopathy
}

National Cancer Institute

\section{Source}

National Cancer Institute. Proximal Tubulopathy. NCI Thesaurus. Code C114477.

A proximal renal tubular disorder resulting in diminished reabsorption of phosphate,

glucose, amino acids, urate, and low molecular weight proteins. 